\title{
New ways to see a smaller world
}

\section{Advances in imaging are allowing researchers to gain better insights into the function of tissues, cells and even individual molecules. Nathan Blow examines the latest technologies lighting the way.}

Competition is often a stimulus for change. But a competition can also be a mirror of a technology's evolution. Take the annual Nikon International Small World Competition and the Olympus BioScapes International Digital Imaging Competition, for example: these events give scientists from around the world the opportunity to submit their best photographed microscopy images for judging by panels of experts.

This year, the winner of the Small World contest, announced in mid-October, was Michael Stringer from Westcliff-on-Sea, UK, for a multicolour, dark-field image of marine diatoms. The winner of the 2008 BioScapes contest was Spike Walker of Penkridge, UK, using Rheinberg illumination of a fairy fly wasp. Entrants in these competitions are using everything from conventional stereomicroscopes to the latest in laser-scanning confocal instrumentation, in some cases with different coloured fluorophores and the newest three-dimensional rendering software, to obtain images ranging from Stringer's diatoms, to a growing bundle of carbon nanotubes, to colourful pictures of individually labelled neurons in a mouse brain.

According to Eric Flem, director of the Nikon competition - now in its 34 th year the size and scope of the contest has grown exponentially in recent years, something he attributes to advances in the underlying imaging technology. "Imaging has become so much easier to do and so much more powerful - it is the norm rather than the exception in most scientific fields these days," he says.

\section{Probing questions}

Researchers often use a little beacon of light, a fluorophore, to see the inside world of the cell. Although a great number and range of fluorescent proteins are currently available for this purpose, improving these to meet the expanding needs of cell biologists can be quite a challenge for developers.

"Often improvements in one characteristic of a fluorescent protein can be reached," says Ilya Kelmanson, head of the product department at fluorescent-protein developer Evrogen in Moscow, "but usually at the expense of another important parameter." An example of this is monomerization of fluorescent proteins, which helps in tagging proteins within the cell, but will generally result in a decrease in the fluorescence intensity. To deal with this, Evrogen often maintains different lines of fluorescent proteins: bright dimeric probes (made of two monomers) used for cell-labelling applications, and less-bright monomeric versions for tagging

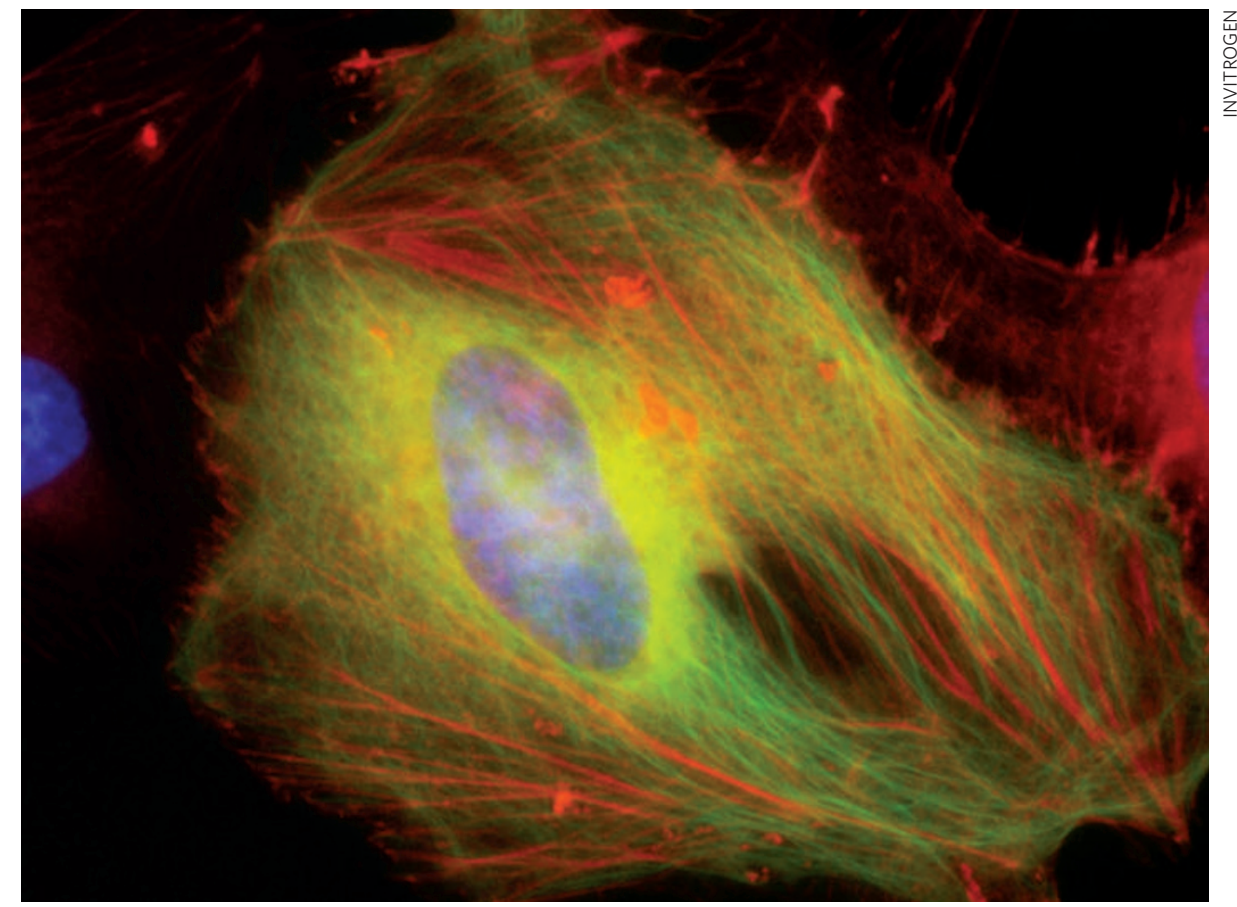

Fluorescent probes and dyes can be used to image the inner world of the cell.

purposes. Kelmanson says that Evrogen is developing a third line of fluorescent proteins that will consist of tandem versions of dimeric fluorescent proteins that have stronger fluorescence than their monomeric counterparts, and good performance and brightness as fusion partners with other proteins.

There are many types of fluorescent proteins that emit in the middle range of the light spectrum - green to yellow. But it is a different story at the far ends of the spectrum. "We still do not have good fluorescent proteins emitting in the near-infrared range," says Kelmanson. Probes in this range could be very useful for whole-body imaging applications and multicolour labelling experiments, he says.

"People are moving away from the blue and the green excitation wavelengths and into the red and near-infrared," says Martin Hoppe, a market manager at microscope developer Leica Microsystems in Wetzlar, Germany. He says that using fluorescent proteins with longer excitation wavelengths helps researchers when it comes to live-cell imaging applications - an area of keen interest for most cell biologists (see 'Light activated') - because these wavelengths tend to be less damaging to cells. Newer dyes have been optimized for near-infrared excitation. One of these, mCherry, one of the 'fruit fluorescent proteins' developed in the lab of Roger Tsien at the University of California,
San Diego - who shared the 2008 Nobel Prize in Chemistry for the discovery and development of green fluorescent protein - is now offered by Clontech in Mountain View, California, along with several fluorescent proteins derived from reef corals.

Developing fluorescent proteins at longer wavelengths, particularly for fluorescence resonance energy transfer (FRET) applications, which are used to measure interactions between two proteins in vivo, "has been a challenge", says George Hanson, a principal scientist working on cell signalling at Life Technologies (formerly Invitrogen) in Eugene, Oregon. Although a challenge, there are potential benefits - FRET efficiency grows and background fluorescence decreases when using longer wavelength fluorophores, according to Kelmanson.

Still, the development of fluorescent proteins that excite at longer wavelengths is only part of the issue for those interested in applying these fluorophores to their FRET studies. Although there are many cyanine to yellow FRET pairs available along with filters and instrumentation preset for reading their interactions, the use of more red-shifted fluorescent protein pairs might take time as optimal parameters and appropriate microscopy hardware and software will need to be developed.

At Life Technologies, Hanson has been working 


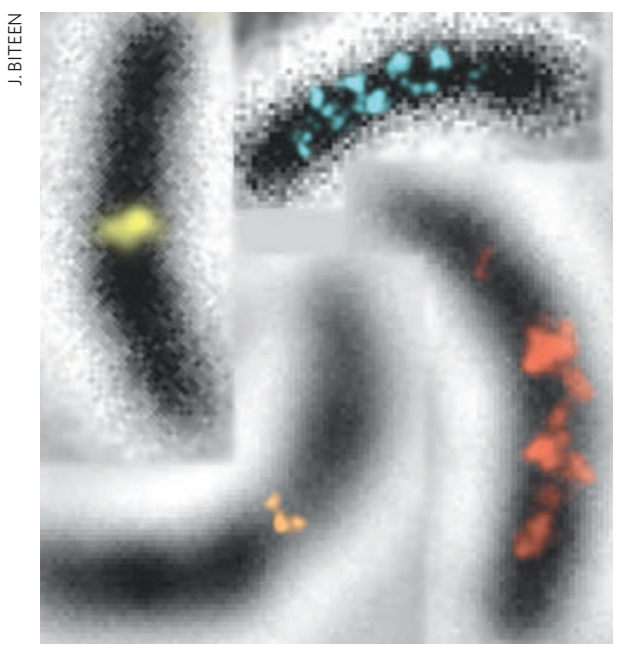

Fluorescence switching is key to single-molecule imaging methods such as PALM and STORM.

to advance the use of FRET in high-throughput screening applications. Here, the challenge of obtaining a strong FRET signal is crucial to overcoming any background fluorescence. Because the simple approach of making a brighter fluorescent protein can be time-consuming, another approach, being used by a number of developers, is to take advantage of the 'states' - the differences between the excitation and emission of a fluorescent protein. Time resolved FRET (TR-FRET) uses long-lived fluorophores that exhibit a time delay between their excitation and emission, minimizing potential interference from background fluorescence. Several companies now offer TR-FRET platforms for cellular interaction assays, including Life Technologies, Cisbio in Bedford, Massachusetts, Covalys of Witterswill, Switzerland, and PerkinElmer located in Waltham, Massachusetts.

\section{Quantum of happiness}

Another route to fluorescence is provided by quantum dots, which "present better photostability than organic dyes and allow for increased multiplexing capability", says Stephen Chamberlain, manager of business development at Life Technologies.

Quantum dots are composed of a semiconductor core shell surrounded by an exterior coating. What makes them unique as fluorophores is their ability to be excited by a single wavelength of light - the emission from a quantum dot is dictated by the size of its core shell. As the size of the core shell can be varied to obtain different light-emission spectra, quantum dots present a particularly interesting solution for researchers interested in experiments using multiple coloured fluorophores.

But unlike fluorescent proteins, quantum dots are not genetically encodable, which has been a stumbling block to more widespread usage. "It is hard to get them into the cell," says Alice Ting, a chemist at the Massachusetts Institute of Technology in Cambridge, who has been working with quantum dots for several years. "And then once inside, how do you target them to a specific protein?"

Most researchers and developers are looking towards the outer coating for targeting. At Life
Technologies, says Chamberlain, they place a polymer coating around the core shell of their Qdot Nanocrystals along with an additional layer of polyethylene glycol on top of the polymer coating to reduce the sticking together of the Qdots, and to provide a surface for the attachment of antibodies and other molecules for targeting.

Despite the challenges of targeting, quantum dots are now being tried in a number of imaging applications. Chamberlain says that like their organic-dye counterparts 20 years ago, researchers are still in the process of developing robust application protocols for quantum dots. Although quantum dots can be used for FRET applications, much like fluorescent proteins, they tend to make better donors in a FRET interaction because they can be excited by light that is blue-shifted relative to their emission. They are being used for in vivo imaging applications, such as looking at blood vasculature, because of their high residence times and ability to excite in the near-infrared region of the spectrum.

Ting says using quantum dots for singlemolecule imaging is a much more straightforward application at the moment, although the issues of delivery and targeting still exist, and several groups are now taking advantage of the greater brightness of quantum dots to track the movement of single molecules within cells.

\section{Microscopes respond}

As the types and range of applications of fluorescent probes and quantum dots continue to grow, developers of confocal microscopes have been working hard to keep instrumentation

\section{LIGHT ACTIVATED}

When scientists first discovered light-absorbing molecules - in microorganisms - that could control the flow of ions into a cell, many researchers became interested in comparing how these proteins differed from their visual pigment counterparts. But Karl Deisseroth at Stanford University in California saw something else in these light-activated channels - a potential tool to dissect the function of the brain.

Deisseroth - along with Edward Boyden, his postdoctoral fellow at the time, now at the Massachusetts Institute of Technology in Cambridge, and his graduate student Feng Zhang - collaborated with microbiologists to hunt for these light-activated channels from various species. "I come from a synaptic physiology background, so we just sequentially tried these and other proteins to find the ones that worked the best," says

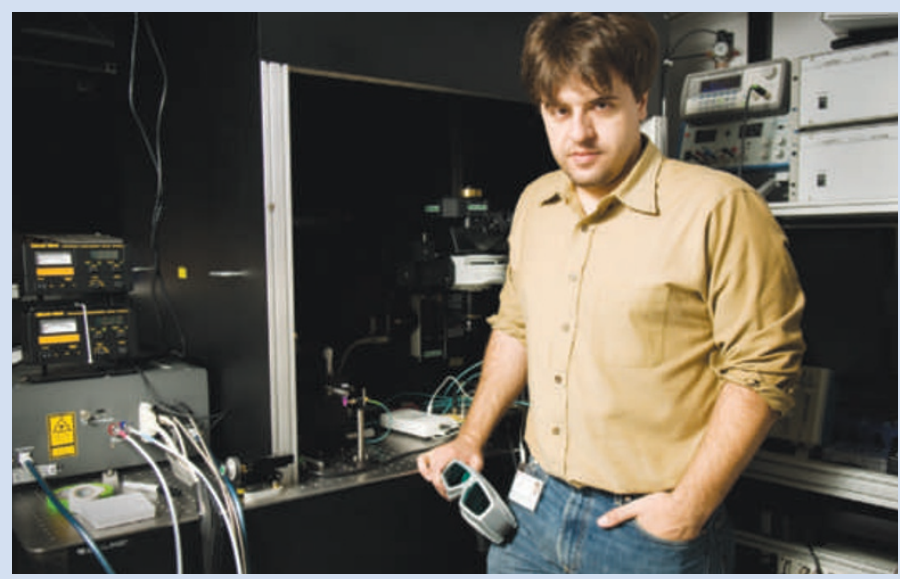

Karl Deisseroth develops optogenetic methods for controlling neural activation.

Deisseroth. It was a collaboration with Georg Nagel and Ernst Bamberg at the Max Planck Institute of Biophysics in Frankfurt, Germany, who supplied a clone of a gene called channelrhodopsin-2 from Chlamydomonas reinhardtii to Deisseroth that opened the floodgates.
Channelrhodopsin-2 is a gated light-sensitive cation channel that uses a molecule of all-trans retinal to absorb photons. When Deisseroth, Boyden and Zhang expressed channelrhodopsin-2 in hippocampal neurons in the mouse brain then shone blue light on the region, they found the cells with channelrhodopsin-2 responded to the light stimulation, opening the channel and initiating the flow of ions, which resulted in an action potential in those neurons ${ }^{6}$. Deisseroth says that one of the most pleasant surprises to emerge from that first series of experiments turned out to be the precision of the system. "Even though these molecules are not designed to generate action potentials and work on this time scale in neurons," he says, "it turns out that they can."

This result led to the start of a new field, coined 'optogenetics' by Deisseroth in 2006, where researchers are combining optics with genetics to explore the workings of neural circuits. In the years since the first description of channelrhodopsin-2, Deisseroth's lab has gone on to advance the system and develop new probes. The team identified and developed two more light-activated 
up to the challenge. "In general, researchers are still requesting the same three things: higher speed, better signal-to-noise and lower toxicity," says Michael Davis, a confocal product manager from Nikon Instruments in Melville, New York. But he is quick to add that there are several emerging fluorescent-protein techniques that have required more sophisticated confocal technology.

Davis says more researchers are now using photoactivatable and photoconvertible proteins in their research. The idea here is to use a specific wavelength of light to excite the fluorescent protein, which can then be turned off, in the case of photoactivatable proteins, or even converted to another colour, in the case of photoconvertible proteins, with a different wavelength of light. Evrogen now offers both kindling red fluorescent protein (KFP-Red), which can be either reversibly or irreversibly activated for either short or long-term protein tracking in a cell, and PS-CFP2, which is a photoconvertible protein that will switch from cyan to green in response to light activation. And MBL International in Woburn, Massachusetts, offers the photoconvertible proteins CoralHue Kaede and CoralHue Kikume, which convert from green to red in response to light activation.

The development of these fluorescent proteins has led to dual scanning systems for confocal microscopes, allowing for the simultaneous imaging and activation of photoactivatable and photoconvertible proteins, along with new spectral imaging technologies that can accurately assay a broad range of possible spectral emissions.

Another trend in the development of confocal instrumentation is the emergence of resonance scanners. "People would like to see the resolution they get out of a point scanner, but with the speed of a field scanner," says Davis. Several developers hope that resonance scanning will bridge this divide. With a resonance-scanning confocal system, the scanner mirrors are moved at extremely high frequencies, permitting much higher frame rates and

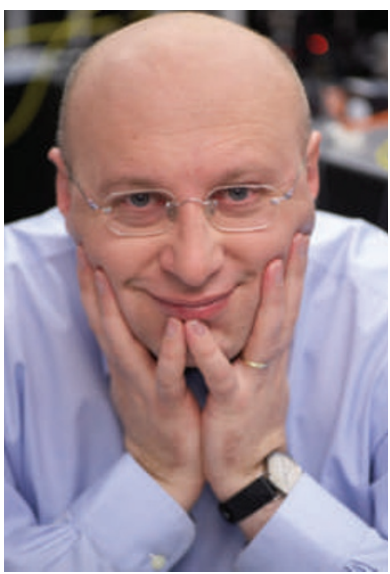

Stefan Hell developed the STED imaging approach.
The ability of a fluorescent protein or a dye to switch states is also at the heart of an emerging field in microscopy. "Fluorescence switching is the common basis to all superresolution methods that have been devised so far," says Stefan Hell, director of the Max Planck Institute for Biophysical Chemistry in Gottingen, Germany, who created a super-resolution imaging method known as stimulated emission depletion (STED) 'nanoscopy'.

Before super-resolution imaging, the resolution limit of optical microscopy was minimizing the time the scanner dwells at any particular point in the sample, allowing researchers to study more dynamic events in a cell while reducing the effects of phototoxicity on it. Both Nikon and Leica Microsystems have introduced resonance-scanning confocal systems in recent years.

\section{Small switches}

Although dual imaging and faster scanning are enhancing fluorescence imaging, the next step in improving the optical resolution of microscopes might require all developers to look more closely at the properties of those fluorescent proteins. "Optics alone can only provide resolution to a certain limit," says Davis. thought to be determined by the diffraction barrier of light. For example, in a highresolution confocal microscope, a beam of light is focused down to a spot on the focal plane of about 200 nanometres in size. Because all molecules within that spot are excited in parallel, the spatial resolution is limited to about this value. Hell's approach with STED was to use the transition states of fluorophores, or their photoswitching capabilities, to effectively reduce the size of that spot - potentially all the way down to a single molecule. In a STED microscope, the focal spot is overlapped with a ring of light that switches off fluorescence everywhere in the spot except at the centre of the ring. The outcome is a much smaller molecules for optogenetic control of neurons. These were an inhibitor called halorhodopsin, and a Volvox channelrhodopsin that can also initiate neuronal activity, but is more than 100 nanometres red-shifted from the peak of the Chlamydomonas channelrhodopsin, allowing separable channels of optical control. They also developed various targeting strategies to get channels expressed in

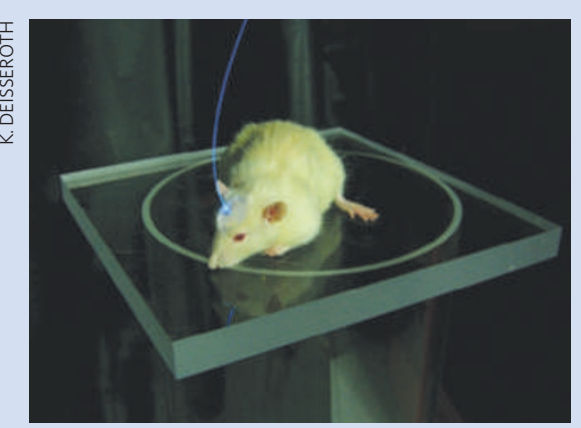

Mouse brain neurons can be stimulated by light from fibre-optic cables attached to the animal. specific neuronal cell types and populations, and fibreoptic/laserdiode hardware for adaptation to mammals.

The growing number of researchers interested in applying optogenetic approaches to their particular research questions has led to an unexpected effort within Deisseroth's lab. "Technology distribution and helping other researchers has been another big part of what we are doing," says Deisseroth. His lab has supplied clones of the channels to more than 300 other labs around the globe for use in organisms ranging from mice to fruitflies. Although the channels have worked in every species tried thus far, in some cases, minor modifications have been required. Although there is sufficient all-trans retinal in the brains of mice for the channels to function properly, invertebrates must be supplemented with all-trans retinal through their diet to achieve channel activation.

As the basic use of these light-activated channels for studying brain function and circuitry gains more traction in the neuroscience community, Deisseroth is taking the technology a step further. He sees patients at Stanford Medical School and, using optogenetic approaches, his lab establishes different animal models of neuropsychiatric disease.

"We now have models of

Parkinson's disease, depression and altered social behaviour relevant to autism," notes Deisseroth. In all of these disorders, the circuit dynamics are not working well, so Deisseroth's goal is to use his optogenetic models to deconstruct

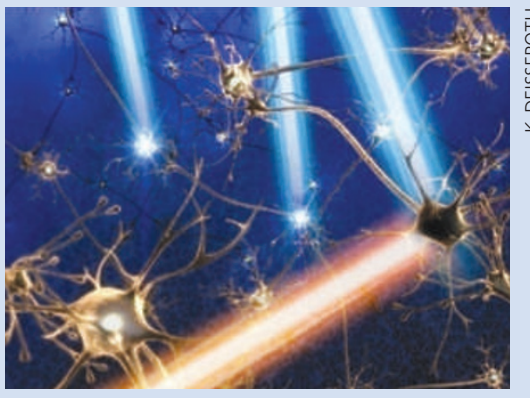

Light is used to open ion channels and activate specific neurons in the brain.

the neural circuitry in an attempt to better understand which parts of the brain are functioning properly, and which are not, in the different disease states.

With the ability to work on a wide range of organisms, an expanding tool kit, and growing interest among researchers, Deisseroth sees even greater possibilities for the system. "In the end, if you can get the gene in, along with the proper light, it works very well." 
fluorescence spot that, when scanned across the specimen, gives super-resolution images automatically.

"STED was the first concept that really showed you could go beyond the barrier of half the wavelength of light in the focal plane," says Hell. More and more super-resolution approaches are starting to be described in scientific journals as researchers see the potential of the method.

For detecting single molecules in cells, William Moerner, a chemist at Stanford University, says photoswitching of single molecules has created a real sense of excitement in the community. By exploiting the ability of fluorophores to switch states, researchers can work with high concentrations of labelling products - crucial for imaging of structures. And, by having most of the labelled molecules 'off' and only a few 'on' at a given moment, they can improve the resolution down to that singlemolecule level of detection.

This premise is at the heart of several newer super-resolution approaches. These include photoactivated localization microscopy $^{1}$ (PALM) developed by Eric Betzig at the Howard Hughes Medical Institute's Janelia Farm Research Campus in Ashburn, Virginia, and his colleagues; fluorescence-PALM, developed by Samuel Hess and his colleagues at the University of Maine in Orono ${ }^{2}$; and stochastic optical reconstruction microscopy ${ }^{3}$ (STORM), developed by Xiaowei Zhuang and her colleagues at Harvard University. These methods can now image structures in cells at a resolution far below 200 nanometres.

Even though fluorophores are used in all these methods, both new and old photoswitchable molecules are proving useful for superresolution imaging. Although Moerner and his colleagues recently used the readily available enhanced yellow fluorescent protein (EYFP) to image proteins at a resolution of less than 40 nanometres in Caulobacter crescentus ${ }^{4}$, he is also involved with the National Institutes of Health
Molecular Imaging Exploratory Center programme in which his group, along with others at Stanford University and Kent State University in Kent, Ohio, are developing new dyes and fluorophores specifically for single-molecule imaging. The centre's efforts are starting to pay off. Researchers there recently invented a new class of photoswitchable molecules based on the switching of an azide functional group to an amine when exposed to activating light beams ${ }^{5}$, as well as covalently linking cyanine dyes to a molecule to improve switching performance in STORM experiments. "Although I know that many groups are working to optimize fluorescent proteins for switching, now the community can work on optimizing small molecules for switching as well," says Moerner.

\section{Higher resolution for all}

For those researchers interested in breaking the diffraction barrier in their own research, the road to super resolution will become easier in the coming years. "Initially, I think there was quite a reluctance to believing that you would get super resolution," says Hell. When he started developing STED microscopy, the initial system relied on a series of flexible lasers, creating a complex system that many outside researchers initially thought would be required for any STED imaging application. But today, Hell says the development of laser systems that are easier to use and have the right light structure to perform STED, alongside ever-improving confocal systems with their spectral-imaging capabilities for those interested in the PALM and STORM approaches, are encouraging researchers to try super-resolution imaging.

Leica Microsystems is the only company

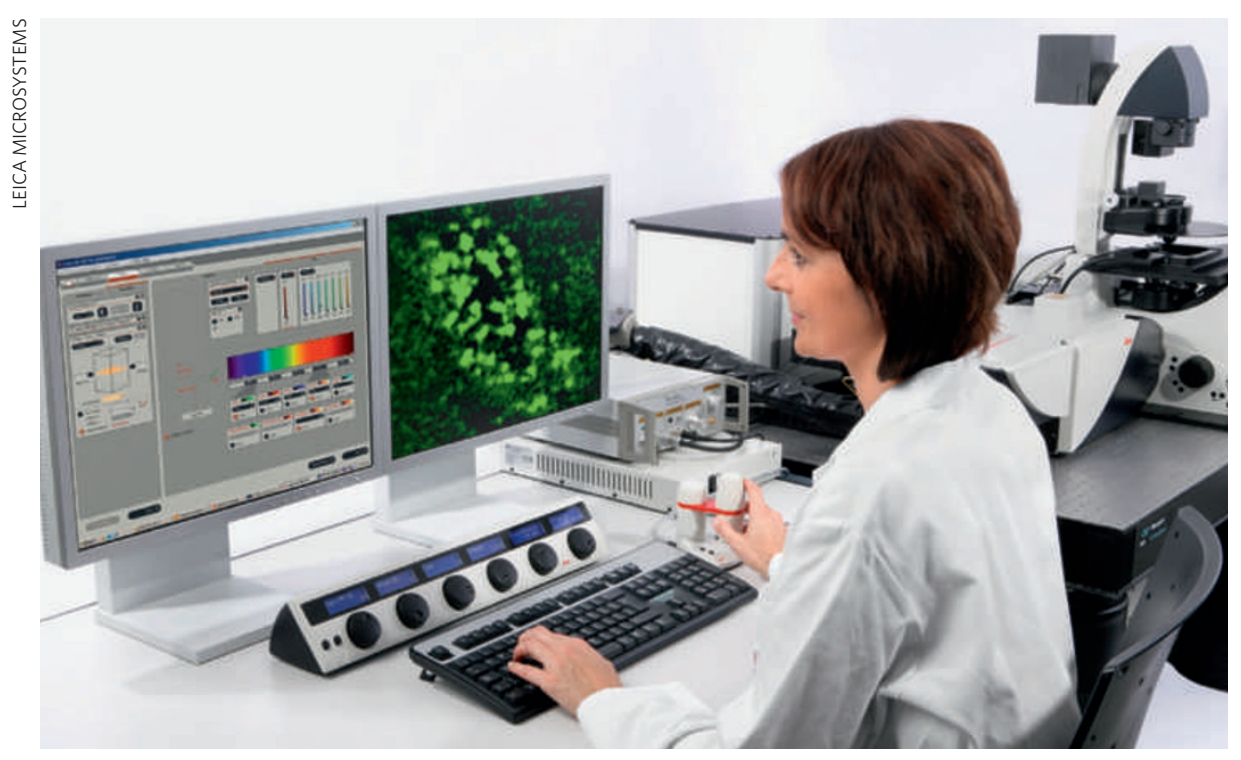

Leica Microsystems recently developed a commercial version of Stefan Hell's STED platform. 